\title{
El aseguramiento de la responsabilidad civil profesional en la práctica de la podología
}

\author{
The assurance of professional liability in practice of the podiatry
}

\author{
Bernardo Perea Pérez ${ }^{(1)}, M^{\text {a }}$ Elena Albarrán Juan ${ }^{(2)}$, Andrés Santiago Sáez ${ }^{(3)}$, \\ Cristina Martín Muñoz ${ }^{(4)}$ \\ ${ }^{(1)}$ Doctor en medicina. Especialista en Medicina Legal y Forense. \\ Profesor Titular Medicina Legal y Forense de la Universidad Complutense de Madrid. \\ Miembro de la Comisión Científica y de Investigación del Colegio de Médicos de Madrid. \\ ${ }^{(2)}$ Doctora en medicina. Especialista en Medicina Legal y Forense. \\ Especialista en Medicina Familiar y Comunitaria. Área 8 Madrid \\ (3) Doctor en medicina. Especialista en Medicina Legal y Forense. \\ Responsable del Servicio de Medicina Legal Hospital Clínico San Carlos. \\ (4) Doctora en medicina. \\ Especialista en Medicina Familiar y Comunitaria. Área 4 Madrid
}

\author{
Correspondencia: \\ Dr. Bernardo Perea Pérez \\ Departamento de Toxicología y Legislación Sanitaria \\ Facultad de Medicina \\ Universidad Complutense de Madrid \\ Plaza Ramón y Cajal s/n \\ 28040 Madrid
}

Fecha de recepción: 14 julio 2009

Fecha de aceptación: 22 diciembre 2009

Los autores declaran no tener ningún tipo de interés económico o comercial.

\section{RESUMEN}

El seguro de responsabilidad civil profesional es un requisito obligatorio para la práctica de la podología en el sector privado, también es muy recomendable tenerlo aunque únicamente se trabaje en la asistencia sanitaria pública. Es un producto asegurador que cubre los daños producidos a pacientes debidos al ejercicio profesional, y también la defensa jurídica en esa circunstancia.

No todos los seguros de responsabilidad civil profesional que hay en el mercado son similares, hay diferencias importantes que pueden condicionar su adecuación a nuestra actividad.

Existen una serie de datos que necesariamente tenemos que conocer sobre nuestro seguro de responsabilidad civil profesional: si es colectivo o individual (el hecho de que se contrate a través de un Colegio Profesional no implica necesariamente que sea colectivo), si tenemos más de un seguro cubriendo la misma actividad, si nuestra sociedad en caso de tenerla está asegurada, si nuestro seguro cubre todas nuestras actividades profesionales, si la cobertura económica es suficiente y si existen franquicias, qué ocurrirá cuando dejemos de trabajar como podólogos, y, por último, asegurarnos de que no existan periodos sin cobertura. Debemos también conocer las exclusiones del seguro, esto es, aquellas circunstancias en las que determinadas actividades no son cubiertas.

Por último, tenemos que saber qué hacer respecto al seguro en caso de reclamación legal o riesgo inminente de que ocurra.

Palabras clave: Seguro de responsabilidad civil profesional; Reclamaciones legales; Malpraxis. 


\begin{abstract}
The assurance of civil professional responsibility is an obligatory requirement for the practice of the chiropody in the private sector, though it is very advisable to have it though only one is employed at the sanitary public assistance. It is an insurance product that covers the damages produced to patients due to the professional exercise, and also the juridical defense in this circumstance.

Not all the assurances of civil professional responsibility that exists on the market are similar, there are important differences that can determine its adequacy to the activity.

There exist a series of information that necessarily we have to know on our assurance of civil professional responsibility: if it is collective or individual (the fact that it is contracted across a Professional College it does not imply necessarily that is collective), if we have more than one assurance covering the same activity, if our society in case of having it is assured, if our assurance covers all our professional activities, if the economic coverage is sufficient and if franchises exist, what will happen when we stop working as chiropodists, and, finally, making sure that should not exist periods without coverage. We have to know also the exclusions of the assurance, this is, those circumstances in which certain activities are not covered.

Finally, we have to know what to do with regard to the assurance in case of legal claim or imminent risk that it happens.
\end{abstract}

Keywords: Assurance of civil professional responsibility; Legal claims; Malpraxis.

Sumario: 1. Introducción. 2. ¿Qué es el seguro de responsabilidad civil profesional?. 3. ¿Qué cubre y qué no cubre el seguro de responsabilidad civil?. 4. ¿Cuándo es necesario?. 5.¿Se deben asegurar las sociedades?. 6. Algunas definiciones básicas. 7. ¿Dónde está la información sobre nuestro seguro de responsabilidad civil?. 8. Aspectos clave a estudiar (y decidir) antes del suscribir un seguro de responsabilidad civil profesional. 9. Algunas exclusiones clásicas en los seguros de responsabilidad civil profesional. 10. Notificación al seguro de responsabilidad civil de los problemas legales. 11. Conclusiones. 12. Bibliografía.

\title{
Referencia normalizada:
}

Perea Pérez B, Albarrán Juan ME, Santiago Sáez A, Martín Muñoz C. El aseguramiento de la responsabilidad civil profesional en la práctica de la podología. Revista Internacional de Ciencias Podológicas 2011; $5(1), 53-61$. 


\section{INTRODUCCIÓN}

El seguro de responsabilidad civil profesional es una necesidad legal y real para el ejercicio de la podología. Legal porque lo exige la ley, como veremos más adelante. Real porque en un contexto de aumento de las reclamaciones legales, trabajar sin seguro es una temeridad (1). Aunque no se han encontrado series publicadas sobre reclamaciones legales contra podólogos en España, el aumento de la presión legal de los pacientes contra los profesionales sanitarios, sin duda también ha debido afectar a esta actividad.

En la Colección de Sentencias Judiciales sobre Responsabilidad Profesional Sanitaria de la Escuela de Medicina Legal de la Universidad Complutense de Madrid, hay siete sentencias correspondientes a podólogos. Teniendo en cuenta que se trata de sentencias de segunda instancia, el número de reclamaciones legales contra podólogos en primeras instancias ha de ser mucho mayor.

La podología, desde la perspectiva del seguro de responsabilidad civil profesional, tiene una serie de peculiaridades que la diferencian de otras actividades sanitarias ${ }^{(1)}$ : la primera es que los tribunales aplican a la podología con cierta frecuencia la obligatoriedad de obtener unos resultados (contrato de obra o resultado) y ésto condiciona a menudo las resoluciones judiciales, la segunda es que la práctica profesional de la podología es básicamente privada, aunque a menudo no ejercida de forma libre sino bajo contrato con entidades mercantiles y la última peculiaridad sería la irrupción en los últimos años de técnicas relativamente agresivas que generan mayor número de demandas con un mayor coste económico ${ }^{(2)}$.

Ante esta situación la necesidad de aseguramiento es ineludible, y generalmente se cumple. Todos los podólogos tienen un seguro y todos lo pagan anualmente, pero prácticamente nadie se ha detenido a estudiar sus detalles y si se adapta o no a sus necesidades concretas ${ }^{(3,4,5)}$.

La póliza del seguro de responsabilidad civil profesional es un documento complejo y difícil de descifrar para los que no están familiarizados con este tipo de cuestiones legales ${ }^{(6)}$.

El objetivo de este título es proporcionar una guía sencilla para entender el seguro de responsabilidad civil, señalar sus característi- cas más importantes y si se adapta o no a nuestra práctica profesional.

\section{2. ¿QUÉ ES EL SEGURO DE RESPONSA- BILIDAD CIVIL PROFESIONAL?.}

El seguro de responsabilidad civil es un producto asegurador que cubre la responsabilidad civil profesional del asegurado frente a terceros derivada de los daños y perjuicios de los que sea responsable causados en el ejercicio de la podología. Esta definición está basada en el artículo 73 de la Ley del Contrato de Seguro (7) que dice: "Por el seguro de responsabilidad civil el asegurador se obliga, dentro de los limites establecidos en la Ley y en el contrato, a cubrir el riesgo del nacimiento a cargo del asegurado de la obligación de indemnizar a un tercero los daños y perjuicios causados por un hecho previsto en el contrato de cuyas consecuencias sea civilmente responsable el asegurado, conforme a derecho" ${ }^{(3,4)}$.

\section{3. ¿QUÉ CUBRE Y QUÉ NO CUBRE EL SEGURO DE RESPONSABILIDAD CIVIL?}

Aunque existen variaciones según el tipo de póliza, todos los seguros de responsabilidad civil profesional cubren al menos las indemnizaciones que se deban pagar a pacientes a consecuencia de daños y perjuicios producidos en el ejercicio de la profesión. También todos cubren los gastos de defensa jurídica.

Algunos seguros ofrecen libertad para la elección de esta defensa jurídica y otros la cubren obligadamente con los servicios jurídicos de la propia aseguradora.

Algunas pólizas también ofrecen un seguro asociado para casos de inhabilitación profesional, aunque este tipo de prestación es discutido en la actualidad ya que estaría primando una situación que es consecuencia de una sanción penal a la que se llega por una actuación profesional no aceptable ${ }^{(4)}$.

El seguro de responsabilidad civil profesional en ningún caso cubre la devolución de honorarios o sanciones económicas en el ámbito penal o administrativo. "Por ejemplo en caso de denuncia penal, el seguro pagaría los gastos de defensa jurídica y la posible indem- 
nización al paciente por los daños y perjuicios ocasionados. No pagaría la eventual devolución de honorarios ni la multa a pagar en caso de que ésta se contemplase en la condena".

Es importante tener presente que el seguro no sólo cubrirá los daños y perjuicios derivados de hechos propios, sino también los de aquellas personas de los que tengamos obligación de responder. Dentro de estos últimos se incluiría el personal auxiliar a las órdenes del asegurado pero no otros profesionales titulados como se verá más adelante.

Habitualmente los seguros de responsabilidad civil profesional también suelen incluir la responsabilidad civil de explotación y patronal del asegurado. De forma básica, la responsabilidad civil de explotación cubre los daños a terceros causados por las instalaciones y la responsabilidad civil patronal cubre los daños personales sufridos por el personal contratado debidos al desempeño de su trabajo.

\section{4. ¿CUÁNDO ES NECESARIO?}

El artículo 46 de la Ley de Ordenación de las Profesiones Sanitarias ${ }^{(8)}$ recoge: "Los profesionales sanitarios que ejerzan en el ámbito de la asistencia sanitaria privada, así como las personas jurídicas o entidades de titularidad privada que presten cualquier clase de servicios sanitarios, vienen obligados a suscribir el oportuno seguro de responsabilidad...que cubra las indemnizaciones que se puedan derivar de un eventual daño a las personas causado con ocasión de la prestación de tal asistencia...".

Por tanto es una obligación legal tener un seguro de responsabilidad civil que cubra nuestra práctica profesional si ejercemos la asistencia privada, la mayoritaria en podología.

En caso de trabajar en exclusiva en el sector público podríamos ejercer sólo bajo la cobertura del seguro de responsabilidad civil del servicio público de salud, pero consideramos que aún así es interesante tener un seguro de responsabilidad civil personal.

La necesidad de disponer de un seguro que cubra nuestra práctica privada se refuerza con el artículo 636 del Código Penal que dice: "Los que realizasen actividades careciendo de los seguros obligatorios de responsabilidad civil que se exigieran legalmente para el ejercicio de aquellas serán castigados con la pena de multa de uno a dos meses".

\section{5. ¿SE DEBEN ASEGURAR LAS SOCIE- DADES?}

Sí. Las sociedades mercantiles o profesionales (salvo alguna excepción muy concreta) tienen entidad jurídica propia, y pueden ser llevadas ante los tribunales de la jurisdicción civil y condenadas, por ello precisan un seguro de responsabilidad civil propio.

Del clausulado de este artículo 46 de la Ley de Ordenación de las Profesiones Sanitarias se deduce la necesidad de aseguramiento de las personas físicas y jurídicas. Esto implica que tienen que asegurarse los podólogos individuales y las sociedades mercantiles que presten asistencia podológica, aunque se diera el caso de que el único accionista de una sociedad fuera un profesional que ya contase con un seguro personal de responsabilidad civil. Respecto a las sociedades profesionales, su obligación de aseguramiento se recoge en el artículo 11.3 de la Ley 2/2007 de Sociedades Profesionales.

\section{ALGUNAS DEFINICIONES BÁSICAS}

Aunque el seguro de responsabilidad civil es un tema complejo, existen algunas definiciones que debemos conocer ${ }^{(9)}$ :

- Entidad aseguradora: Es la entidad obligada a responder por el asegurado cuando se materialice el riesgo cubierto. Puede ser una compañía mercantil o una mutua (agrupación de profesionales).

- Tomador del seguro: Es la persona o entidad que contrata con la compañía aseguradora la cobertura del riesgo. En caso de seguros contratados individualmente, suele corresponderse con el propio asegurado. En caso de seguros colectivos, el tomador suele ser un colegio profesional.

- Asegurado: Podólogo que tiene cubierta la responsabilidad civil derivada de su actividad profesional por la compañía aseguradora.

- Beneficiario: Perjudicado que sufre el daño y al que resarce la entidad aseguradora en nombre del asegurado. 
7. ¿DÓNDE ESTÁ LA INFORMACIÓN SOBRE NUESTRO SEGURO DE RESPONSABILIDAD CIVIL?

La información sobre el seguro está en un documento llamado "póliza del seguro". Esta póliza suele tener al menos una sección de condiciones generales y otra de condiciones particulares (en ocasiones también existen apéndices, etc).

Las condiciones generales suelen ser comunes a todas las pólizas de igual tipo (de hecho suelen ser comunes con las del resto de profesiones sanitarias). Las condiciones particulares son las realmente importantes ya que en ellas se recogen las cláusulas específicas negociadas. En el caso relativamente frecuente de contradicción entre las condiciones generales y las particulares prevalecerán siempre estas últimas. Es, sobre todo, en las condiciones particulares donde debemos buscar las características de nuestro seguro.

La póliza del seguro suele estar en poder del tomador del seguro, en caso de seguros individuales generalmente el propio asegurado, y en caso de seguros colectivos, habitualmente el Colegio. Los podólogos que tienen seguro colectivo recibirán un certificado de adhesión a la póliza.

\section{ASPECTOS CLAVE A ESTUDIAR (Y DECIDIR) ANTES DEL SUSCRIBIR UN SEGURO DE RESPONSABILIDAD CIVIL PROFESIONAL}

Son múltiples los aspectos que hay que tener presente en un seguro de este tipo, por ello es muy recomendable que cualquier elección o cambio esté adecuadamente asesorado; no obstante, y a modo de introducción, hemos seleccionado los aspectos que consideramos esenciales:

\subsection{Elección entre aseguramiento indivi- dual o colectivo.}

El aseguramiento colectivo suele implicar que existe un tomador distinto del propio podólogo (habitualmente un colegio profesional), y que dicho podólogo se adhiere a este seguro. Esto implica que es un seguro general, adaptado a la actividad de la mayoría de los podólogos y cuyo grado de adaptación a unas circunstancias concretas es limitado.

Los otros rasgos del seguro colectivo son claramente ventajosos. En primer lugar suele ser un seguro asesorado, vigilado por los tomadores y, por lo tanto, con menor posibilidad de problemas de cobertura. En segundo lugar, la capacidad de presión sobre la compañía aseguradora (llegado el caso) no es igual en el caso de un asegurado individual que en el caso de un seguro colectivo (frecuentemente con miles de asegurados detrás). Y, por último, y debido a una mera cuestión de número, las primas de los seguros colectivos suelen ser mucho más económicas que las de los seguros individuales.

El seguro individual tiene como principal ventaja la posibilidad de adaptarlo a nuestras circunstancias personales, y el anonimato total respecto a los siniestros que pudiesen acaecer.

\subsection{Cantidad económica cubierta.}

A este respecto es importante tener en cuenta tres aspectos:

- Cantidad económica cubierta.

- Número de siniestros cubiertos.

- Existencia o no de franquicias.

Habitualmente en la práctica podológica, donde los daños producidos suelen ser muy limitados, las cantidades habituales manejadas por los seguros (de 150.000 a $300.000 €$ actualmente) son más que suficientes.

Es importante saber si esta cantidad se refiere a cada uno de los posibles siniestros que pudiésemos tener, o a la suma de todos los siniestros anuales. Aunque repetimos que este problema no suele ser frecuente en podología.

Por último es importante saber si existen franquicias. Es raro que existan para daños personales, pero son frecuentes para daños materiales (que en muchas ocasiones son una parte importante de los gastos de un seguro de responsabilidad civil de este tipo).

\subsection{Periodo de cobertura del seguro.}

Es éste un punto fundamental, posiblemente el más importante ${ }^{(10)}$.

En general, y sin entrar en especificaciones técnicas, existen tres tipos de seguros: 
- "Claim made”: Aseguran los siniestros (reclamaciones legales) declarados durante el año de vigencia de la póliza, independientemente del año en el que se hubieran producido los tratamientos que los provocaron. Habitualmente, una vez transcurrido el año asegurado (y si no hay renovación de la póliza), el seguro ya no respondería de las reclamaciones legales que se produjeran posteriormente.

- "Claim ocurrance": Aseguran los siniestros debidos a tratamientos ocurridos durante el año asegurado, aunque el problema legal se hubiese planteado posteriormente. Estos seguros no cubren siniestros (reclamaciones legales) ocurridos durante el año asegurado si el tratamiento hubiese sido previo.

- Formas mixtas.

A nuestro criterio las formas mixtas, generalizando, son las más adecuadas. Los tipos "claim made" y "claim ocurrance" plantean cada uno sus limitaciones, pero lo verdaderamente peligroso es cambiar de un tipo de seguro a otro sin el adecuado asesoramiento ya que puede provocar la aparición de un "período ventana" (período temporal en el cual no hay cobertura de seguro). "Por ejemplo, si durante el año 2008 tuvimos un seguro tipo "claim made" y en el 2009 hemos cambiado a un seguro tipo "claim ocurrance", si se produjese en el año 2009 una demanda judicial por un tratamiento ocurrido durante el año 2008 estaríamos sin cobertura, y esto es lo más peligroso que puede ocurrir",

Existen dos factores más a tener en cuenta respecto a la cobertura temporal: el tipo de cobertura temporal que tenía nuestro seguro anterior (para evitar los períodos "ventana" antes señalados) y la cobertura en el momento de jubilación o cese de actividad. Hay que ser conscientes de que el cese de actividad no impide ser demandado por la consecuencia de nuestros tratamientos previos. La responsabilidad civil contractual prescribe a los quince años (a contar desde que el perjudicado conoce el daño), por lo tanto es posible tener una reclamación judicial después de jubilado. Pudieran darse incluso casos de reclamaciones civiles a herederos una vez fallecido el podólogo. Por ello es muy importante conocer la cobertura del seguro en este período.

\subsection{Concurrencia de seguros.}

Entendemos por concurrencia de seguros la existencia de dos seguros distintos para cubrir el mismo riesgo. Es una situación a evitar ya que no reporta ningún beneficio al asegurado y puede generar problemas derivados de la falta de coordinación entre los seguros concurrentes. No obstante, existe una situación en la que es difícil evitar esta concurrencia, sería el caso de una práctica profesional mixta pública-privada. En esta situación el seguro de responsabilidad civil contratado para cubrir, en exceso de ciertas cantidades indemnizatorias, la asistencia privada también podría cubrir determinadas situaciones ocurridas durante el ejercicio en la sanidad pública. Esto es, el seguro de la sanidad pública cubriría el primer tramo, y el segundo tramo a partir del máximo de cantidad cubierta por el seguro anterior, estaría cubierto por el seguro de responsabilidad civil privado.

Creemos recomendable que todos los seguros profesionales que nos conciernan, personales profesionales o de sociedades profesionales, estén suscritos con la misma compañía o mutua aseguradora lo que facilita mucho los trámites en caso de siniestro.

\subsection{Objeto del seguro.}

Es también fundamental que el objeto del seguro (en este caso la actividad profesional cubierta) se adapte a nuestras circunstancias particulares. Si no es así puede haber problemas en caso de reclamación legal.

Nuestros seguros suelen cubrir las indemnizaciones y defensa jurídica derivada de daños y perjuicios económicos causados a terceros por errores profesionales en el ejercicio de la profesión de podólogo. La profesión de podólogo está delimitada según la Ley de Ordenación de las Profesiones Sanitarias (art. 7.2.d), "los Diplomados universitarios en Podología realizan las actividades dirigidas al diagnóstico y tratamiento de las afecciones y deformidades de los pies, mediante las técnicas terapéuticas propias de su disciplina" y el Real Decreto 649/1988. Todo lo que quede fuera de esta definición no estará asegurado. 
Si nuestra práctica profesional, por tener una doble titulación o por cualquier otra causa, se saliese de estos límites, tendríamos que ampliar la actividad asegurada.

\section{ALGUNAS EXCLUSIONES CLÁSICAS EN LOS SEGUROS DE RESPONSABILI- DAD CIVIL PROFESIONAL.}

A continuación recogemos diversas exclusiones que suelen contemplarse en la generalidad de nuestros seguros de responsabilidad civil profesional ${ }^{(11)}$, aunque debamos leer atentamente el clausulado de nuestra póliza porque existen peculiaridades. "Un ejemplo es la exclusión en algunas pólizas de las maniobras clínicas, que precisando la realización de un documento de consentimiento informado, se realizan sin él".

\subsection{Fabricación, elaboración o venta de} productos farmacéuticos, especialidades propias, productos medicinales, etc.

Este hecho es una constante, no sólo en todos los clausulados de los seguros, sino también en el resto de la legislación sanitaria.

En tanto no varíe el marco legislativo actual, está excluida la responsabilidad civil derivada de la prescripción de medicamentos ${ }^{(12)}$. También está excluida la responsabilidad civil derivada de la fabricación, elaboración o venta de cualquier producto "medicinal" (incluyendo desde verdaderas sustancias farmacológicas, a preparados de plantas medicinales o medicamentos "alternativos"). Estas sustancias requieren un control estricto por parte de la Agencia Española del Medicamento, con seguros específicos de responsabilidad civil.

Respecto a las ortesis podológicas, que tienen la consideración de productos sanitarios a medida, la Ley de Garantías y Uso Racional del Medicamento y Productos Sanitarios recoge en su disposición decimotercera lo siguiente: "La colocación o puesta en servicio de productos sanitarios a medida por un facultativo, en el ejercicio de sus atribuciones profesionales, no tendrá la consideración de dispensación, comercialización, venta, distribución, suministro o puesta en el mercado de los mismos, a los efectos de los artículos 3.1 y 101. En todo caso, el facultativo deberá separar sus honorarios de los costes de fabricación”.

\subsection{Procedimientos curativos "no consa- grados".}

En este término tan ambiguo incluimos todos aquellos procedimientos alternativos que no hayan sido aceptados por las instituciones profesionales o científicas. Realmente es algo difícil de determinar ya que la línea entre procedimientos con una efectividad probable (acupuntura) y otros que rayan la charlatanería (cromoterapia) no es muy nítida. El consejo, es que si alguien realiza algún procedimiento de este tipo, lo comuniqué al seguro de responsabilidad civil para intentar su inclusión en la cobertura de la póliza ${ }^{(13)}$.

\subsection{Ensayos clínicos.}

La normativa que regula los ensayos clínicos con seres humanos es muy estricta. Entre los requisitos necesarios figura la existencia de un seguro específico de responsabilidad civil que cubra los posibles riesgos del ensayo ${ }^{(14)}$. El seguro profesional de práctica clínica no es válido para estas situaciones.

\subsection{Utilización de procedimientos prohibi- dos o en fase de experimentación.}

Los procedimientos prohibidos no son cubiertos por los seguros. Aquí también se incluiría la utilización de productos farmacéuticos o sanitarios no autorizados o cualquier medicamento o material que carezca de los preceptivos permisos (ya sea de la Agencia Española del Medicamento o de la EMEA).

\subsection{Actos dolosos o derivados del incumpli- miento voluntario de normas legales, éticas o profesionales.}

Respecto a los actos dolosos, no hay duda. Cualquier comportamiento que intencionadamente intente dañar al paciente no puede ser cubierto por un seguro de responsabilidad civil profesional.

La segunda parte del enunciado es más problemática. Es evidente que los incumplimientos voluntarios no deben ser cubiertos, pero en ocasiones puede ser difícil diferenciarlos de los incumplimientos negligentes (por olvido). "Un ejemplo claro de ello es la falta de un 
documento de consentimiento informado por escrito en determinadas situaciones".

Otra posible consecuencia de este enunciado, sería la falta de cobertura en caso de trabajar en centros sanitarios sin las correspondientes licencias administrativas.

\subsection{Reclamaciones dirigidas contra otro profesional titulado, aún cuando trabaje a las órdenes del asegurado.}

Esta exclusión es importante. En caso de que un profesional autónomo (con su correspondiente seguro de responsabilidad civil profesional) establezca un contrato de prestación de servicios con otro profesional autónomo (por ejemplo otro podólogo), cualquier demanda motivada por la actuación de este segundo profesional deberá ser cubierta por su propio seguro.

\subsection{Reclamaciones derivadas de daños me- ramente estéticos.}

En general, los tratamientos sanitarios con finalidad estética son excluidos de los seguros de responsabilidad civil profesional. Otro asunto diferente es que las técnicas curativas o rehabilitadoras utilizadas tengan también un resultado estético. En cualquier caso, si decidimos emplear procedimientos con finalidad primordialmente estética, debemos consultar su cobertura al seguro de responsabilidad civil.

\section{NOTIFICACIÓN AL SEGURO DE RESPONSABILIDAD CIVIL DE LOS PROBLEMAS LEGALES.}

Tenemos obligación, por ley (art. 16 de la Ley de Contrato del Seguro), de notificar los siniestros (en este caso reclamaciones legales) a la compañía aseguradora en un plazo de siete días desde que hayamos tenido conocimiento, salvo que se haya pactado otro plazo. Aunque bien es cierto que ésto no suele tomarse en sentido estricto, salvo auténticas dejaciones por parte del profesional.

Ante esta obligación surgen algunas cuestiones:

\section{1. ¿Qué debemos notificar?.}

En ocasiones los profesionales no saben si deben notificar una amenaza o deben esperar a tener la notificación formal del juzgado. Ni una cosa, ni la otra. En primer lugar, es razonable consultar, en caso de duda, a los servicios jurídicos colegiales o a los de la propia compañía aseguradora. Ante cualquier solicitud de documentación motivada por un desencuentro con un paciente es razonable consultar. Y siempre debe hacerse en caso de recibir notificación formal de solicitud de información por parte de terceros (abogados de los pacientes, servicios públicos de inspección o consumo, comisiones deontológicas, etc.), y por supuesto en caso de notificación judicial informando de la existencia de una denuncia o demanda.

\section{2. ¿A quién se debe notificar?.}

Depende del clausulado de la póliza y de las circunstancias concretas. En principio se podría notificar al tomador del seguro o a la propia entidad aseguradora.

\section{CONCLUSIONES.}

A la vista de todo lo anterior, es evidente la necesidad de que cualquier podólogo tenga un seguro adecuado de responsabilidad civil profesional, y que lo conozca. En caso de tener un seguro colectivo (cuyo tomador suele ser un colegio profesional) se debe conocer, sobre todo, la cobertura económica y temporal, y si se adapta al tipo de actividad profesional realizado. En caso de tener un seguro individual, además de lo anterior se debe elegir la cuantía económica cubierta y estudiar el clausulado de la póliza cada vez que se renueve (habitualmente cada año). Cualquier cambio de una póliza de responsabilidad civil a otra debe ser asesorado para evitar períodos sin cobertura. Por último, en caso de tener un problema legal con un paciente que implique la intervención de terceros (oficinas municipales de información al consumidor, comisiones deontológicas, abogados, etc.), se debe notificar este hecho al seguro inmediatamente (Tabla 1). 
Tabla 1. Aspectos a estudiar en las pólizas de responsabilidad civil profesional podológica (por orden de importancia):

1. Adecuación a nuestra actividad profesional (en caso de duda consultar con la compañía aseguradora).

2. No existencia de periodos sin cobertura (asunto crítico en los cambios de seguro de responsabilidad civil).

3. Estudio de la cobertura después del cese de actividad o jubilación.

4. Existencia o no de libertad de elección de defensa jurídica.

5. Adecuación de la cantidad económica asegurada y existencia o no de franquicias.

6. Concurrencia de diferentes seguros que pudiéramos tener contratados (sobre todo en caso de ejercer simultáneamente la práctica pública y privada).

7. Actividades excluidas de la cobertura del seguro.

\section{BIBLIOGRAFÍA}

1. Marín m. Responsabilidad profesional. Rev. Esp. Podología. 2004; XV (5):68-70.

2. Carles M. Responsabilidad por una práctica médica inadecuada: una perspectiva económica. Gac. Sanit. 2003; 17(6):494-503.

3. Lorenzo R, Megía I. El seguro de responsabilidad civil de profesionales de la sanidad. En: Lorenzo R, editor. Responsabilidad legal del profesional sanitario. Madrid: Ed. Edicomplet, 2000.

4. Fernández JM. Sistema de responsabilidad médica. Granada: Ed. Comares, 2000.

5. Peran J. La responsabilidad civil y su seguro. Barcelona: Ed. Tecnos, 1998.

6. Ruiz A. Apuntes sobre la responsabilidad civil profesional en el sector sanitario. Revista Española de Seguros.2003; 114:119-132.

7. Ley 50/1980, de 8 de octubre, de contrato de seguro. (Acceso 24 de abril de 2009). Disponible en: http://www.boe.es/aeboe/consultas/bases_datos/doc.php?coleccion=iberlex\&id=1980/22501

8. Ley 44/2003, de 21 de noviembre, de ordenación de las profesiones sanitarias. (Acceso 24 de abril de 2009). Disponible en: http://www.boe.es/boe/dias/2003/11/22/pdfs/A41442-41458.pdf

9. Greco O. Diccionario de Seguros. Barcelona: Valleta Ed. 2004.

10. Núñez JP. Delimitación temporal de las coberturas de responsabilidad civil. Calidad y Riesgo 2007; 6:85-88.

11. Hernández A. Contrato de seguro: Exclusión de cobertura y cláusulas limitativas. Barcelona: Ed. CEDECS, 1998.

12. Becerro de Bengoa R.. Prescripción y receta podológica. El Peu 2002; 22(1):8-17.

13. Martínez-Nova A. Terapias complementarias. El Peu 2003;23(2):76-81

14. Jiménez L. El seguro obligatorio de ensayos clínicos: riesgos, límites y cláusulas de exclusión. Actualidad del derecho sanitario. 2007; 139:447-453. 